\title{
Selectivity for Interaural Time Difference in the Owl's Midbrain
}

\author{
Terry Takahashi and Masakazu Konishi \\ Division of Biology 216-76, California Institute of Technology, Pasadena, California 91125
}

\begin{abstract}
The barn owl uses the interaural difference in the timing of sounds to determine the azimuth of the source. When the sound has a wide frequency band, localization is precise. When localizing tones, however, the barn owl errs in a manner that suggests that it is confused by phantom targets. We report a neural equivalent of these phenomena as they are observed in the spacespecific neuron of the owl's inferior colliculus. When stimulated with a tone, the space-specific neuron discharges maximally at interaural time differences (ITDs) that differ by one period of the stimulus tone. Changing the stimulus frequency changes the period of the ITD-response functions, but 1 ITD evokes, in most neurons, a maximal response, regardless of frequency. This ITD is the characteristic delay (CD). When stimulated with noise, there is a maximal response only to ITDs at or near the CD. Thus, the space-specific neuron can unambiguously signal the $\mathrm{CD}$, provided that the signal contains more than 1 frequency. The preferential response to a single ITD, which is observed with noise stimuli, was also observed when the summed waveform of the best frequency and another tone, F2, was presented. The response of the space-specific neuron to these 2-tone stimuli could not be accounted for by the summing or averaging of the ITD-response functions obtained with the best frequency or F2 alone, suggesting that nonlinear neural processes are involved.
\end{abstract}

For the barn owl, the interaural differences in the timing and intensity of sounds are the primary cues for the horizontal (azimuthal) and vertical (elevational) coordinates of the source respectively (Knudsen and Konishi, 1979; Moiseff and Konishi, 1984). Interaural time difference (ITD), with which the present report shall deal exclusively, results when a sound source is displaced to one side of the midline and the sound waves reach one ear before they do the other. ITD has 2 components: transient disparity and phase disparity. Transient disparity is the difference in the arrival times of the first wavefront. Phase disparity is the difference in phase of each frequency component in a sound. Behavioral studies suggest that the barn owl is able to derive ITD from the interaural phase differences of all frequency components in a signal (Moiseff and Konishi, 1981).

When a sound contains a broad range of frequencies, the owl localizes the source precisely. When a sound is composed of only 1 frequency, the owl errs in a manner that suggests that its auditory system is unable to match unambiguously a given cycle of the sound in one ear with the corresponding cycle in the other ear. Thus, to the owl, the "real" ITD, which depends solely on the lateral displacement of the source and is independent of frequency, and "phantom" ITDs, which differ from the real ITD by 1 period of the stimulus tone, are equivalent and represent

Received May 24, 1985; revised May 27, 1986; accepted June 6, 1986.

${ }^{1}$ We thank Drs. Andrew Moiseff, W. E. Sullivan, and Hermann Wagner for their helpful comments. This work was supported by NIH Grant NS14617 (M.K.) and NIH Postdoctoral Fellowship NS06825 (T.T.)

Correspondence should be addressed to $\mathrm{T}$. Takahashi at the above address.

Copyright (c) 1986 Society for Neuroscience $0270-6474 / 86 / 123413-10 \$ 02.00 / 0$ possible locations of the source. A phase ambiguity is said to exist (see also Konishi, 1973; Mills, 1972). If the sound contains more than 1 frequency, each frequency channel in the owl's auditory system will have associated with it a number of ITDs that differ by 1 period of the channel's frequency. Importantly, however, the "real" ITD will be common to all frequency bands. The auditory system can resolve phase ambiguity by accessing the information in multiple frequency channels.

Early neurophysiological studies demonstrated that neurons in the cat inferior colliculus discharge maximally at ITDs that differ by 1 period of the stimulus frequency (Geisler et al., 1969; Rose et al., 1966). Notably, one ITD, which was termed the characteristic delay (CD), evokes a similar relative discharge level regardless of the frequency. CD, like the "real" ITD mentioned above, is thus represented in all frequency channels contributing to the passband of the neuron. One might expect, therefore, that sound source locations can be unambiguously represented by the activity of single neurons that have a CD. However, neurons of the cat's inferior colliclus show a cyclic response even when stimulated with wide-band stimuli such as noise (Geisler et al., 1969). They are phase-ambiguous. Thus, although it is possible to show under experimental conditions that a neuron has a CD, higher centers cannot read the CD from the activity of that neuron.

We present evidence below to suggest that space-specific neurons of the external nucleus of the barn owl's inferior colliculus (ICx of Knudsen, 1983) are able to signal the CD by virtue of inhibitory and excitatory interactions between frequency channels. Selectivity for $C D$ by nonlinear interactions is a new phenomenon in the study of binaural neurons.

\section{General properties of the space-specific neuron}

Space-specific neurons, originally termed "limited field cells" by Knudsen and Konishi (1978), are found in the external nucleus of the barn owl's inferior colliculus. They can be driven by a noise emanating only from a sharply circumscribed location in space or by the combination of interaural time and intensity difference that would be generated by a noise source at that location (Knudsen and Konishi, 1978; Moiseff and Konishi, 1981). They cannot be excited by monaural stimuli. Their frequency tuning curves are generally broad (Knudsen, 1984; Knudsen and Konishi, 1978), although a best frequency can usually be discerned. The best areas of their receptive fields are generally insensitive to changes in absolute sound intensity and to the type of stimulus, i.e., noise, tones, or clicks (Knudsen and Konishi, 1978; Moiseff and Konishi, 1981). Finally, the spacespecific neuron's selectivity for 1 interaural cue remains constant for all effective values of the other cue, suggesting that there is no trading between time and intensity (Moisefr and Konishi, 1981; Takahashi et al., 1984).

\section{Materials and Methods}

Adult barn owls (Tyto alba) of both sexes $(n=15)$ were anesthetized by intramuscular injections $(5 \mathrm{mg} / \mathrm{hr}$ ) of ketamine hydrochloride (Vet- 
alar, $100 \mathrm{mg} / \mathrm{ml}$; Bristol Laboratories) and inserted into a custom-built stereotaxic device that held the head such that the plane containing the center of the ear bars and the ventral surface of the palatine ridge was at $45^{\circ}$ from horizontal (beak below ear bar). A stainless steel plate was cemented onto the skull and the stereotaxic device removed. Owls were generally used for more than 1 recording session, and the plates allowed us to reestablish the same head angle each time an owl was used. After the head was secured in the correct orientation, head feathers were plucked, a small incision was made in the scalp, and the underlying skull was opened with rongeurs. The dura was kept moist with silicon grease (Dow-Corning) throughout an experiment. After an experiment the craniotomy was resealed with dental cement, and the scalp was sutured. A topical, antibacterial cream (Neosporin, Burroughs-Wellcome) was applied to the edges of the wound before the owl was returned to its cage.

We isolated space-specific neurons $(n=129)$ from the external nucleus of the inferior colliculus using glass-insulated platinum-iridium microelectrodes. Electrode tracks were histologically verified. Extracellularly recorded action potentials were amplified and discriminated on the basis of their amplitudes (Ortec Window Discriminator, model 730). The output from the discriminator was stored on a hard disk by a PDP $11 / 40$ computer as the time of the occurrence of action potentials relative to a synchronizing pulse.

In earlier experiments, we used a calibrated sound delivery system described previously (Moiseff and Konishi, 1981, 1983). Briefly, the opening of a magnetic earphone was connected to an externally threaded stainless steel cylinder $7 \mathrm{~mm}$ in diameter. The cylinder was screwed into a matching stainless steel ring sutured into the owl's ear canal Sound pressure levels (SPL) near the eardrum were measured with a General Radio 1900 Wave Analyzer in conjunction with a $12.5 \mathrm{~mm}$ microphone (Breuel \& Kjaer) connected to a $1.2 \mathrm{~mm}$ diameter probe tube surgically implanted near the eardrum. In these experiments, the noise stimulus consisted of bursts of band-passed pseudorandom noise (center frequency $7 \mathrm{kHz}, 15 \mathrm{~dB} /$ octave rolloff). In later experiments, we used an improved sound delivery system in which the earphone and microphone probe tube had a common opening close to the eardrum. In this system, sound pressure and spectral levels, and frequency and phase responses of the earphones were measured with a Data Precision Data 6000 digital signal analyzer in conjunction with a Knowles BT1752 microphone with calibrated probe tube. The amplitude of spectral components in the noise stimulus used in these experiments did not fluctuate by more than $6 \mathrm{~dB}$ between 3 and $8 \mathrm{kHz}$. The earphones, which were used in both earlier and later experiments, did not differ in phase or amplitude response by more than $7 \mu \mathrm{sec}$ (at $6667 \mathrm{~Hz}$ ) and $4 \mathrm{~dB}$ (between 3000 and $9000 \mathrm{~Hz}$ ), respectively. The frequency response of each earphone was essentially flat: It showed no more than $4 \mathrm{~dB}$ fluctuations in the $3000-9000 \mathrm{~Hz}$ range. Changing the stimulus delivery system did not affect the neuronal properties under study.

Auditory stimuli consisted of flat and filtered spectrum noise bursts, tone bursts, or 2-tone bursts, all of which were $100 \mathrm{msec}$ in duration and had $5 \mathrm{msec}$ rise and decay times. Each stimulus was presented at a rate of $1.5 / \mathrm{sec}$ and repeated 5-20 times. The level and timing of the stimuli in the ears could be controlled independently by manual or digital attenuators and a computer-controlled, microsecond interaural time shifter. Space-specific neurons respond only when a stimulus contains an appropriate combination of interaural time and level difference. The choice of a level difference within the effective range of level disparities does not affect a space-specific neuron's selectivity for ITD (Moiseff and Konishi, 1981; Takahashi et al., 1984). All stimuli were delivered at the interaural level difference that evoked the highest discharge rate. The best interaural level difference for any 1 cell generally remained constant, regardless of the type of stimulus or the frequency of the tone burst.

For comparing responses to tones and noise, tone and noise bursts were usually matched for root-mean square (RMS) sound pressure levels. In some experiments, the spectral level of a particular frequency band (bandwidth $=24.4 \mathrm{~Hz}$ ) in noise was matched to that of a tone burst at the frequency corresponding to the center frequency of the band of intcrest. Two-tone complexes were produced by summing the outputs of 2 independent oscillators (XR 2206, Exar) and introducing the summed waveform to the time shifter. The levels of the 2 frequencies at the output of the earphone were equal. Because the 2 oscillators were independent, the phase relationship of the 2 tones varied constantly.

The best frequency (BF) of a neuron, i.e., the frequency that evoked the greatest spike discharge, was determined by listening to the response of the cell to different frequencies on an audio monitor or by plotting the number of spikes against frequency. Initially, both methods were used and similar results were obtained. In later experiments, BFs were determined by the first method only.

\section{Calculation of $C D$}

In order to determine the $C D$ of a neuron, we first plotted the response of a neuron as a function of the ITD of a stimulus tone. The plots are referred to as "delay curves." ITDs were varied from +300 to -300 $\mu \mathrm{sec}$ or from +150 to $-150 \mu \mathrm{sec}$, usually in $30 \mu \mathrm{sec}$ steps. Below, positive ITDs indicate that the stimulus in the right ear led; negative ITDs indicate that the stimulus in the left ear led. We chose, as standard test frequencies, tones the periods of which were multiples of $30 \mu \mathrm{sec}$. Thus, we most commonly tested neurons with $3333,3704,4167,4761,5555$, 6667 , and $8333 \mathrm{~Hz}$ tone bursts.

As an initial estimate of $C D$, delay curves obtained with different tones were normalized, superimposed, and inspected for an ITD that evoked the same relative discharge level regardless of frequency. In addition, for neurons with bandwidths broad enough to be stimulated with 3 or more test frequencies, we applied circular statistics for the calculation of CD (Kuwada and Yin, 1983; Yin and Kuwada, 1983). For this procedure, ITD was first converted into interaural phase (IPD). Because the ITD range over which we collected data encompasses more than 1 period of the tones we used, each IPD value recurs. The response of a neuron was therefore averaged at each IPD. The averaged response was treated as a vector having a magnitude $r$ (spikes) and a direction IPD (cycles). The $x$ and $y$ components of the vector are defined as

$$
\begin{aligned}
& x=r \cos \text { IPD, } \\
& y=r \sin \text { IPD. }
\end{aligned}
$$

A mean interaural phase (MP) was then determined by summing $x$ and $y$ components and evaluating the arctangent of the ratio between summed $y$ and summed $x$. Thus,

$$
\mathrm{MP}=\arctan \left(\sum y / \sum x\right) .
$$

MP values were corrected for phase mismatch of the earphones by adding or subtracting a correction value determined for each earphone pair at frequencies the periods of which differed by $10 \mu \mathrm{sec}$. Alternatively, we first obtained a delay curve, reversed the earphones while holding the neuron, and derived a second delay curve. MP values calculated for the 2 curves were averaged. The largest correction value obtained by the 2 methods was $7 \mu \mathrm{sec}$ (at $6667 \mathrm{~Hz}$ ). For closer examination of delay curves, we also sampled at $10 \mu \mathrm{sec}$ intervals. MP values calculated from delay curves obtained at $10 \mu \mathrm{sec}$ intervals differed by no more than 0.03 cycles (average 0.007 cycles; 49 delay curves; 11 neurons) from the values calculated from $30 \mu \mathrm{sec}$ delay curves. This was true for test frequencies as high as $8333 \mathrm{~Hz}$ (Fig. 1).

The corrected MP values were plotted as a function of frequency (MP vs frequency plot or phase-frequency plot), and a regression line was derived. The line has the equation

$$
\mathrm{MP}=\mathrm{CD} \times \mathrm{F}+\mathrm{CP},
$$

where $F$ is the stimulus frequency, and the slope of the line is the $C D$. The $y$-intercept, or characteristic phase (CP), is an index of the relative response level at the $\mathrm{CD}$. If, at the $\mathrm{CD}$, the spike level is maximal, $\mathrm{CP}$ has a value of 1.0 or, equivalently, 0 cycles. If the response is minimal, $\mathrm{CP}$ assumes a value of 0.5 cycles. Values between 1.0 (or 0 ) and 0.5 cycles indicate that the cell discharges at intermediate levels when the CD is presented (Yin and Kuwada, 1983).

The goodness of fit of the data to the regression line was determined by calculating the mean square error (MSE) between observed and expected values. The significance level was read from a graph published by Yin and Kuwada (1983, their Fig. 11B). A level of $p<0.05$ was used as the criterion for linearity.

\section{Interpolation of peak position}

A delay curve is a plot of spike counts (normalized or unnormalized) as a function of ITD obtained at a sampling interval of 10 or $30 \mu \mathrm{sec}$. Segments of higher discharge rates can be recognized on a delay curve. Such segments recur cyclically, typically in curves obtained with tonal 


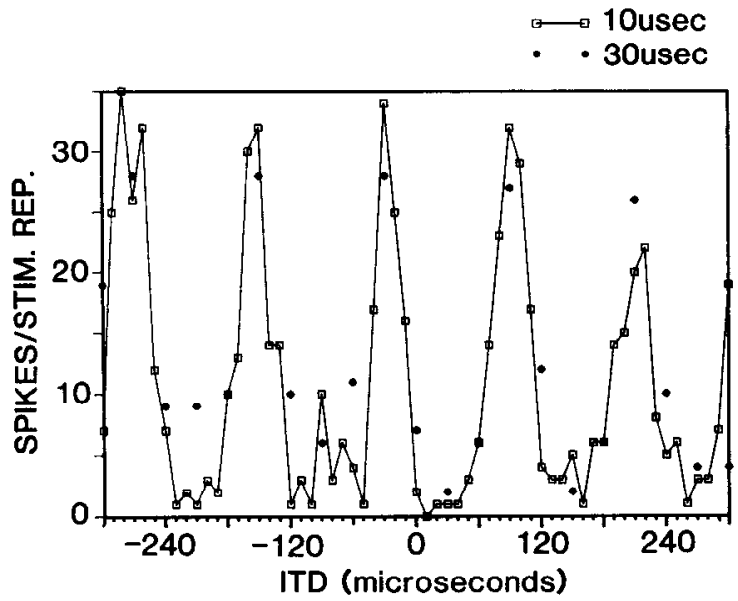

Figure 1. Sampling of selectivity for ITD at 10 and $30 \mu \mathrm{sec}$ intervals. The results obtained with $10 \mu \mathrm{sec}$ intervals (open squares) and $30 \mu \mathrm{sec}$ intervals (filled, unconnected circles) yield similar delay curves. The $M P$ obtained with the $10 \mu \mathrm{sec}$ steps is 0.74 cycles; that obtained with the $30 \mu \mathrm{sec}$ steps is 0.75 cycles. The stimulus frequency was $8333 \mathrm{~Hz}$. Tone burst level $=46 \mathrm{~dB}$ SPL in left and right ears. Unit is from the right external nucleus.

stimuli, or they occur once, as in some curves obtained with noise stimuli (see below). Whichever the case, the mean of ITDs (mean peak position, or mean ITD) in such a segment is a good estimate of the ITD that would elicit a maximal discharge. The mean was calculated as follows:

$$
\text { Mean ITD }=\sum_{i=1}^{n} R_{i} \times \mathrm{ITD}_{i} / \sum_{i=1}^{n} R_{i}
$$

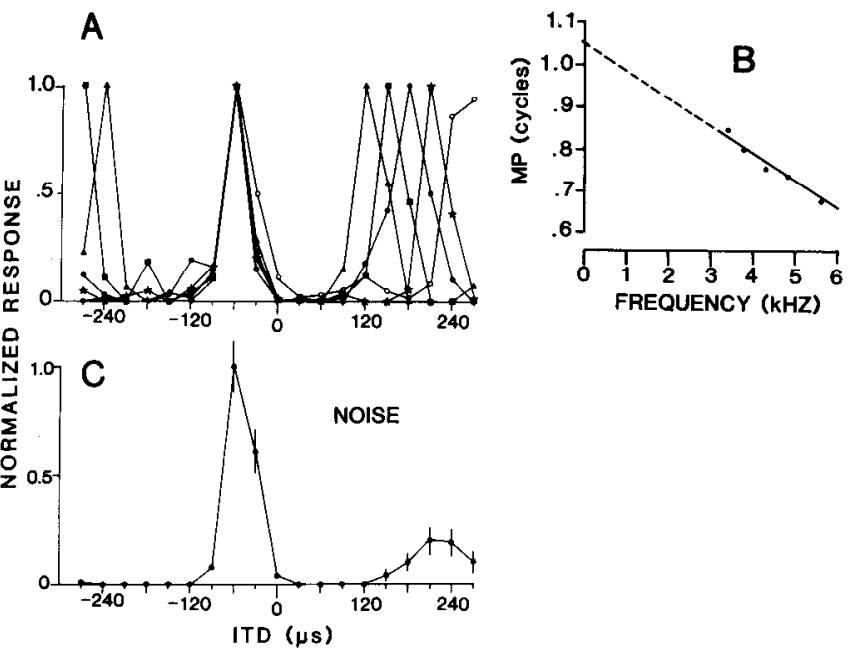

Figure 2. Determination of CD. A, Delay curves of a space-specific neuron (right external nucleus) obtained with tonal stimuli (in Hz): 3333 (open circles), 3704 (stars), 4167 (filled circles), 4761 (squares), and 5555 (triangles). The levels of all tone bursts in left and right ears were 56 and $51 \mathrm{~dB}$ SPL, respectively. The periodicities of the curves are equal to those of the stimulus tones. However, at $-60 \mu \mathrm{sec}$, all curves reach a common maximum ("peak"). This value is an estimate of the neuron's CD. $B$, Plot MP vs frequency (phase-frequency plot) obtained from the data shown in $A$. The slope of the line, which represents CD, is -65 $\mu \mathrm{sec}$, and the $y$-intercept, or characteristic phase (CP), is 1.05 cycles [mean square error (MSE) $=0.0007, p<0.01$ ]. $C$, Response of the neuron shown in $A$ to noise. The levels of noise burst in left and right cars werc 56 and $51 \mathrm{~dB}$ SPL (RMS), respectively. With noisc stimuli only, a single "primary" peak occurs at $-60 \mu \mathrm{sec}$, which is near the CD calculated from the MP vs frequency plot shown in $B$. Vertical lines through data points represent the SDs.
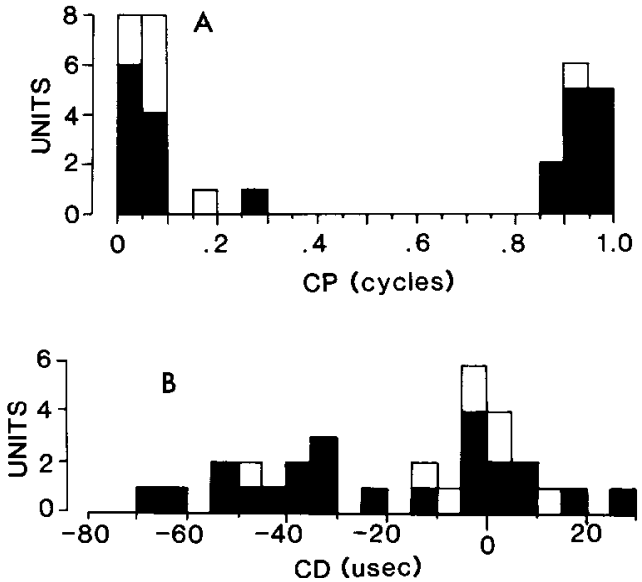

Figure 3. Distribution of $\mathrm{CP}(A)$ and $\mathrm{CD}(B)$. The histograms are based on 31 neurons. Solid bars represent the neurons whose MP vs frequency plots were linear at the $p<0.05$ level $(n=23)$. Neurons that did not fit this criterion are represented by open bars $(n=8)$. The distribution of CP suggests that space-specific neurons generally discharge at nearmaximal levels at the $C D$.

where $R_{\mathrm{i}}$ is the spike number at the $i$ th ITD and $n$ is the number of sample ITDs.

The method above assumes that segments of high response are symmetrical about the mean ITD. If they are, calculation of mean peak position over any number of ITDs above and below the ITD observed to evoke the highest discharge rate should yield the same mean ITD. We calculated the mean peak position for $30 \mathrm{high}$-response segments over a range extending 30 and $90 \mu \mathrm{scc}$ above and below observed peak ITDs. Regression of the mean peak positions calculated over a \pm 30 $\mu \mathrm{sec}$ range against values calculated over a $\pm 90 \mu \mathrm{sec}$ range yielded a line having a correlation coefficient of 0.99 (slope $=1.02, y$-intercept $=$ 0.09 ).

\section{Results}

Response to tonal stimuli: the characteristic delay

The discharge of a space-specific neuron to tonal stimuli is a cyclic function of ITD. Delay curves have periodicities corresponding to the period of the stimulus tone burst. It can be seen

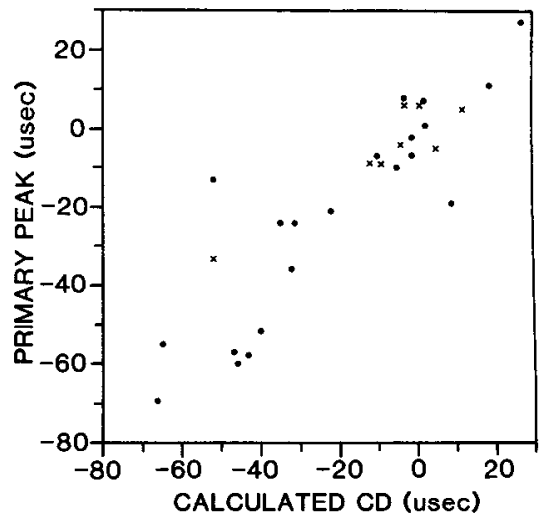

Figure 4. Correlation between the calculated $\mathrm{CD}$ and the mean position of primary peaks. The mean peak position (see Materials and Methods) was calculated over a $90 \mu \mathrm{sec}$ range above and below the most effective ITD observed ( $n=29$ neurons). The regression line has a slope of 0.86 , a $y$-intcrcept of $6.0 \mu \mathrm{sec}$, and a correlation coefficient of 0.87 . Solid circles represent neurons in which MP vs frequency plots were linear at the $p<0.05$ level; $\times s$ represent neurons in which MP vs frequency plots did not meet this criterion. 


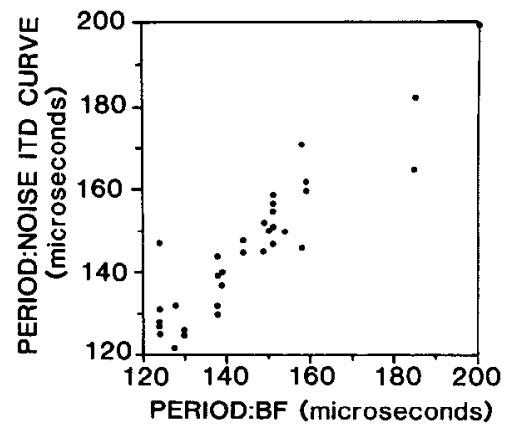

Figure 5. Correlation between the period of a neuron's BF and the period of its noise delay curve. The latter was obtained by calculating the difference between mean ITDs (see Materials and Methods). The regression line has a slope of 0.86 , a $y$-intercept of $21 \mu \mathrm{sec}$, and a correlation coefficient of 0.91 .

in Figure $2 A$ that when, for example, a $5555 \mathrm{~Hz}$ tone was presented, the curve (triangles) displayed local maxima (peaks) every $180 \mu$ sec of delay; i.e., at $-240,-60$, and $+120 \mu \mathrm{sec}$. The same could be said for the $4761,4167,3704$, and $3333 \mathrm{~Hz}$ tones, which resulted in delay curves having periodicities of 210,240 , 270 , and approximately $300 \mu \mathrm{sec}$, respectively.

It is clear from Figure $2 A$ that all curves reach a maximal level of discharge at $-60 \mu \mathrm{sec}$ regardless of stimulus frequency. This ITD is a good estimate of the CD. For 31 neurons that could be tested with more than 3 frequencies, we applied circular statistics (Yin and Kuwada, 1983) to calculate CD and CP (i.e., the relative discharge level of a neuron at the $C D)$. Of these 31 neurons, $23(74 \%)$ showed a linear relationship between MP and frequency at a significance level of $p<0.05$.

An example of the results of this analysis is shown in Figure $2 B$. The data plotted are from the neuron in Figure $2 A$. The slope of the line (CD) is $-65 \mu \mathrm{sec}$, and its $y$-intercept (CP) is 1.05 cycles. These values are in good agreement with the values expected from inspection of the normalized delay curves $(C D=$ $-60 \mu \mathrm{sec}, \mathrm{CP}=1.0$ ). The distributions of $\mathrm{CP}$ and $\mathrm{CD}$ values for the 31 neurons that were analyzed by circular statistics are shown in Figure 3, $A$ and $B$, respectively. The tendency of spacespecific neurons to discharge maximally at the CD may be seen in the distribution of CP. Of the 31 neurons, $27(87 \%)$ attained firing levels within $10 \%$ of maximum at the $C D$ (i.e., between 0 and 0.1 or between 0.9 and 1$)$ and $30(97 \%)$ attained firing levels within $20 \%$ of maximum (i.e., between 0 and 0.2 or between 0.8 and 1 ). Of the 23 neurons for which the phase-frequency distribution was linear $(p<0.05), 20(87 \%)$ discharged at levels within $10 \%$ of maximum and $22(96 \%)$ discharged at levels within $20 \%$ of maximum. The distribution of $C D$ values obtained shows that our sample is skewed towards relatively small values. (The maximum ITD experienced by the owl is $180 \mu \mathrm{sec}$.)

\section{Response to noise}

When the stimulus was a noise burst (filtered or flat spectrum) rather than a tone burst, space-specific neurons typically displayed delay curves having a single "primary" peak accompanied by smaller "secondary" pcaks at ITDs removed from that of the primary peak by integral multiples of the period of its best frequency. An example is shown in Figure $2 C$, which illustrates the response of the neuron of Figure $2 A$ to a noise burst. In the experiments described in this section, noise and tone bursts were matched for RMS levels. (Effects of matching spectral levels are described in the next section.) A strong response was seen at $-60 \mu \mathrm{sec}$, which is near the calculated CD, and a weaker response (about 20\%) was seen between +180 and

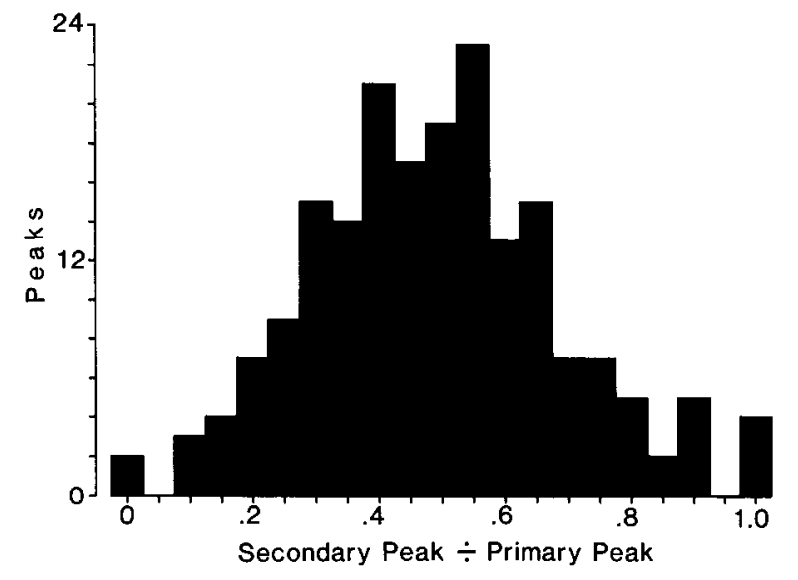

Figure 6. Distribution of the sizes of secondary peaks relative to the height of the primary peak. The data were taken from 119 neurons $(n=$ 188 peaks; some neurons had more than 1 secondary peak). Mean = $0.47, \mathrm{SD}=0.20$.

$+270 \mu \mathrm{sec}$, which is where one would expect a secondary peak to occur, considering that the neuron's BF was $3.8 \mathrm{kHz}$ (period = $263 \mu \mathrm{sec}$ ).

Noise delay curves were available for 29 of the 31 neurons for which we calculated CD using circular statistics. Of the 29 neurons, $26(90 \%)$ displayed primary peaks within $15 \mu \mathrm{sec}$ of the calculated CD. At the $30 \mu \mathrm{sec}$ interval with which the delay curves of these neurons were obtained, the accuracy is $\pm 15 \mu \mathrm{sec}$. The mean position (see Interpolation of Peak Position, above) of the primary peak and its correlation with the calculated value of CD are shown as a scatter diagram in Figure 4 . The correlation suggests that the primary peak corresponds to the CD.

The period of a noise delay curve is correlated with a neuron's BF. The relationship is shown as a scatter diagram in Figure 5. Only neurons that had clear secondary peaks and unambiguous BFs were included for this analysis $(n=33)$. Periodicities of noise delay curves were obtained by calculating the difference between mean ITDs (see Interpolation of Peak Position, above).

A distribution of secondary peak height relative to primary peak height is shown in Figure 6. The distribution, taken from 119 neurons tested with flat or filtered noise (some neurons had more than 1 secondary peak), had a mean of $0.47(\mathrm{SD}=0.21)$. The mean secondary peak height (with respect to primary peak height) did not differ significantly whether the noise stimulus had a flat or filtered spectrum ( $p>0.07, t$ test; filtered spectrum noise: 101 cells, 144 peaks, mean secondary peak height $=0.48$, $\mathrm{SD}=0.21$; flat spectrum noise: 18 cells, 44 peaks, mean secondary peak height $=0.44, \mathrm{SD}=0.14$ ).

From the normalized plot of Figure $2 C$, it can be seen that with noise, space-specific neurons respond preferentially to a unique ITD. Nonnormalized delay curves showed that noise bursts at the ITD of secondary peaks elicited a lower response in some cells than did a BF tone burst at the same ITD. This effect was termed secondary peak suppression. In other cells, noise bursts at the ITD of the primary peak elicited a higher response than did a BF tone burst at the same ITD. This effect was termed primary peak facilitation.

An example of sccondary pcak suppression is shown in Figurc $7 A$. When presented with its $\mathrm{BF}(8100 \mathrm{~Hz}$, solid line $)$, the neuron discharged maximally ( 20 spikes/stimulus presentation) at -30 and $+90 \mu \mathrm{sec}$. When stimulated with a noise burst (dotted line), the discharge at $+90 \mu \mathrm{sec}$ declined to $38 \%$ of that recorded using the BF. Noise had little effect on the discharge rate at $-30 \mu \mathrm{sec}$. Secondary peak suppression was observed in $28 \%$ of our sample of 74 neurons. The neuron shown in Figure $7 B$ is an example of facilitation of the response at the ITD of the primary peak 

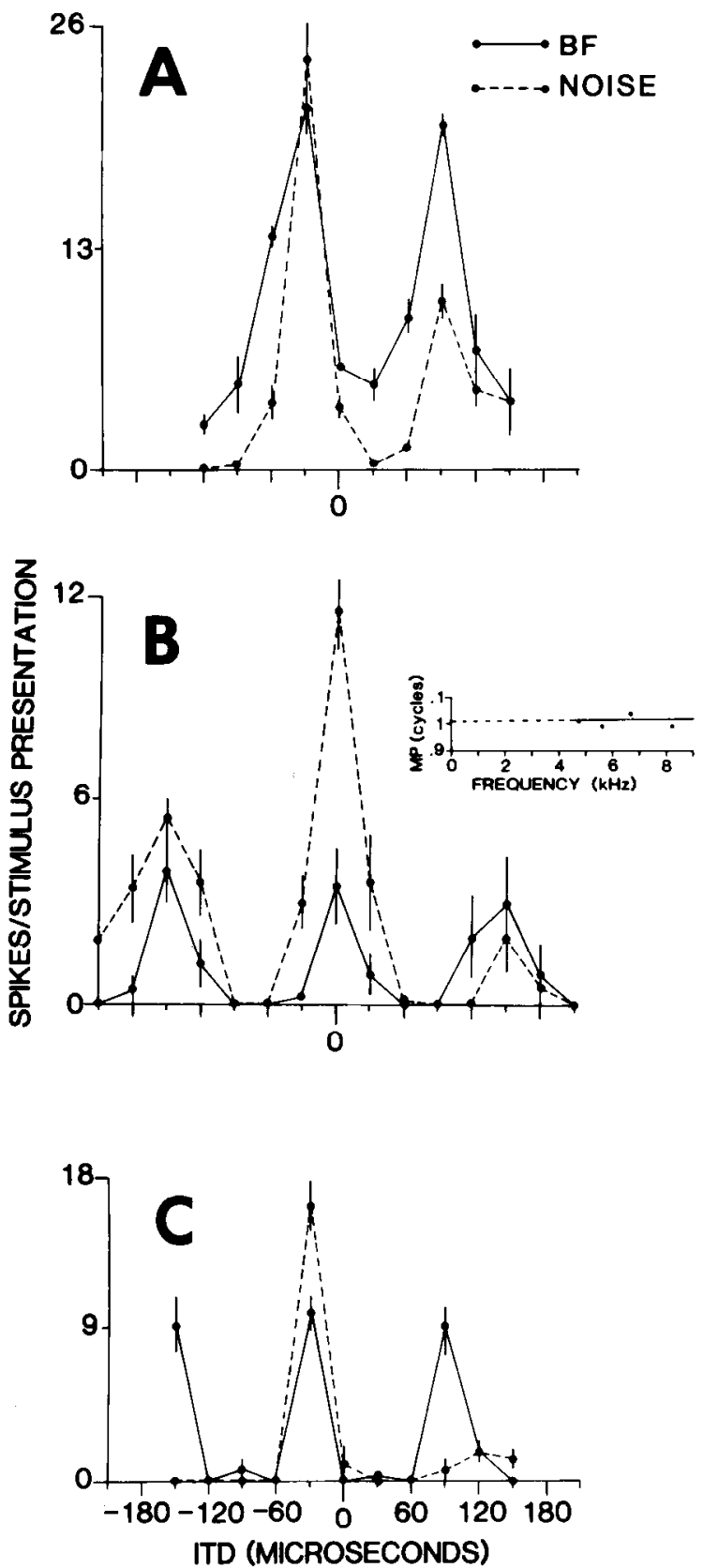

Figure 7. Noise and BF delay curves. Nonnormalized delay curves of 3 space-specific neurons obtained with the BF (solid line) and noise (dashed line). Noise and tone bursts were matched for RMS levels. $A$, Noise suppresses the response to the ITD of the secondary peak compared with the response evoked by the BF $(8100 \mathrm{~Hz})$. The RMS noise level and BF burst was $46 \mathrm{~dB}$ SPL in left and right ears. Unit from right external nucleus. $B$, Noise enhances the response at the $C D$ (see phasefrequency plot in inset; $\mathrm{CD}=1.6 \mu \mathrm{sec}, \mathrm{CP}=0.01$ cycles, $\mathrm{MSE}=0.0002$, $p<0.01)$ to levels above that obtained with the BF $(6900 \mathrm{~Hz})$. RMS level of noise and BF bursts was $56 \mathrm{~dB}$ SPL in left and right ears. Unit from left external nucleus. $C$, Noise facilitates the response at the ITD of the primary peak and suppresses the response at the ITD of the secondary peak relative to the response levels observed by stimulation with the BF $(7800 \mathrm{~Hz})$. The RMS noise level and BF bursts was $56 \mathrm{~dB}$ SPL in left and right cars. Unit from right external nucleus.

without a suppression of the response at the ITD of the secondary peak. In this neuron, the CD was found to be equal to $1.6 \mu \mathrm{sec}$ (inset, Fig. $7 B$ ), which is near $0 \mu \mathrm{sec}$, the ITD of the primary peak (mean ITD of primary peak $=1.4 \mu \mathrm{sec}$ ). When

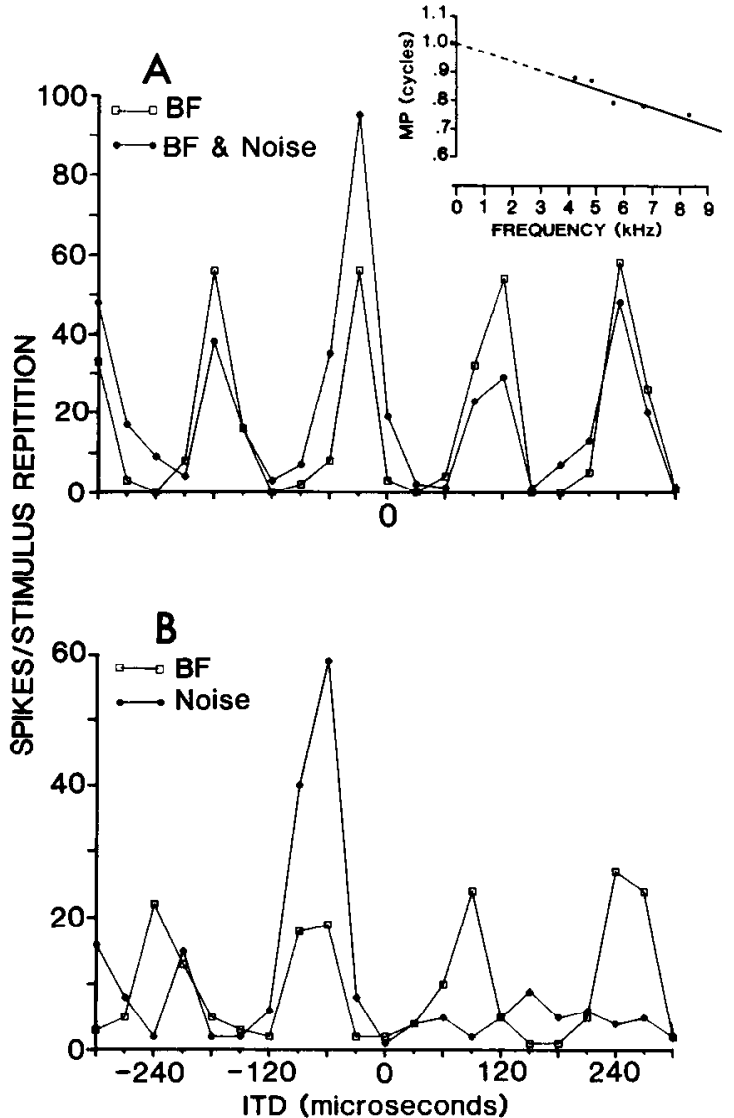

Figure 8. Effects of matching spectral levels. The spectral level of the $\mathrm{BF}$ component in noise stimuli was matched to that of a tone burst at the BF. $A$, Tone burst at the $\mathrm{BF}(6667 \mathrm{~Hz})$ was delivered along with a noise burst [noise burst level $=45 \mathrm{~dB}$ SPL (RMS) in both ears]. The delay curve thus obtained (filled circles) is superimposed on that obtained with a tone burst at $\mathrm{BF}$ alone (open squares). The spectral level of the BF component in the noise and tone burst was $46 \mathrm{~dB}$ SPL in each ear. The presence of noise facilitates the response to $-30 \mu \mathrm{sec}$ (mean ITD of primary peak $=-35.9 \mu \mathrm{sec}$ ), the $\mathrm{CD}$ (see phase-frequency plot in inset; slope $=-32.2 \mu \mathrm{sec}, y$-intercept $=1.0, \mathrm{MSE}=0.0007, p$ $<0.01$ ), whereas it suppresses the response to -180 and $+120 \mu \mathrm{sec}$, ITDs that evoke responses equal to that of the $C D$ when the stimulus is a tone. $B$. RMS level of the noise stimulus was increased so that the spectral level of the $\mathrm{BF}$ component $(6250 \mathrm{~Hz})$ matched that of a tone burst at BF. Thus, the spectral level of the BF component in the noise and tone burst was $30 \mathrm{~dB}$ SPL in each ear. The response to noise at $-30 \mu \mathrm{sec}$ is triple the response to the BF at the same ITD. At other ITDs, the response to noise is generally less than the response to the $B F$. Neurons in $A$ and $B$ from right external nucleus.

stimulated with a tone burst at its $\mathrm{BF}(6900 \mathrm{~Hz}$, solid line), the neuron discharged at similar levels at the CD (3.5 spikes/stimulus presentation) and at -150 and $+150 \mu \mathrm{sec}(3.9$ and 3.0 spikes/stimulus presentation, respectively). When stimulated with a noise burst, its response at $0 \mu \mathrm{sec}$ was more than triple (11.6 spikes/stimulus presentation) that observed with the BF. Primary peak facilitation was observed in $36 \%$ of our sample of 74 neurons. An example of a neuron showing both suppression and facilitation ( $36 \%$ of our sample of 74 neurons) is shown in Figure $7 \mathrm{C}$. At $-30 \mu \mathrm{sec}$, the response of this neuron to noise was nearly double that to the best frequency $(7800 \mathrm{~Hz}$ ) (noise: 16 spikes/stimulus presentation; BF: 9 spikes/stimulus presentation). At the ITD of the secondary peaks, the responses to noise were $0 \%$ (at $-150 \mu \mathrm{sec})$ and $18 \%$ (at $+90 \mu \mathrm{sec}, 1.6 \mathrm{spikes} /$ stimulus presentation) of that to its $\mathrm{BF}$. 


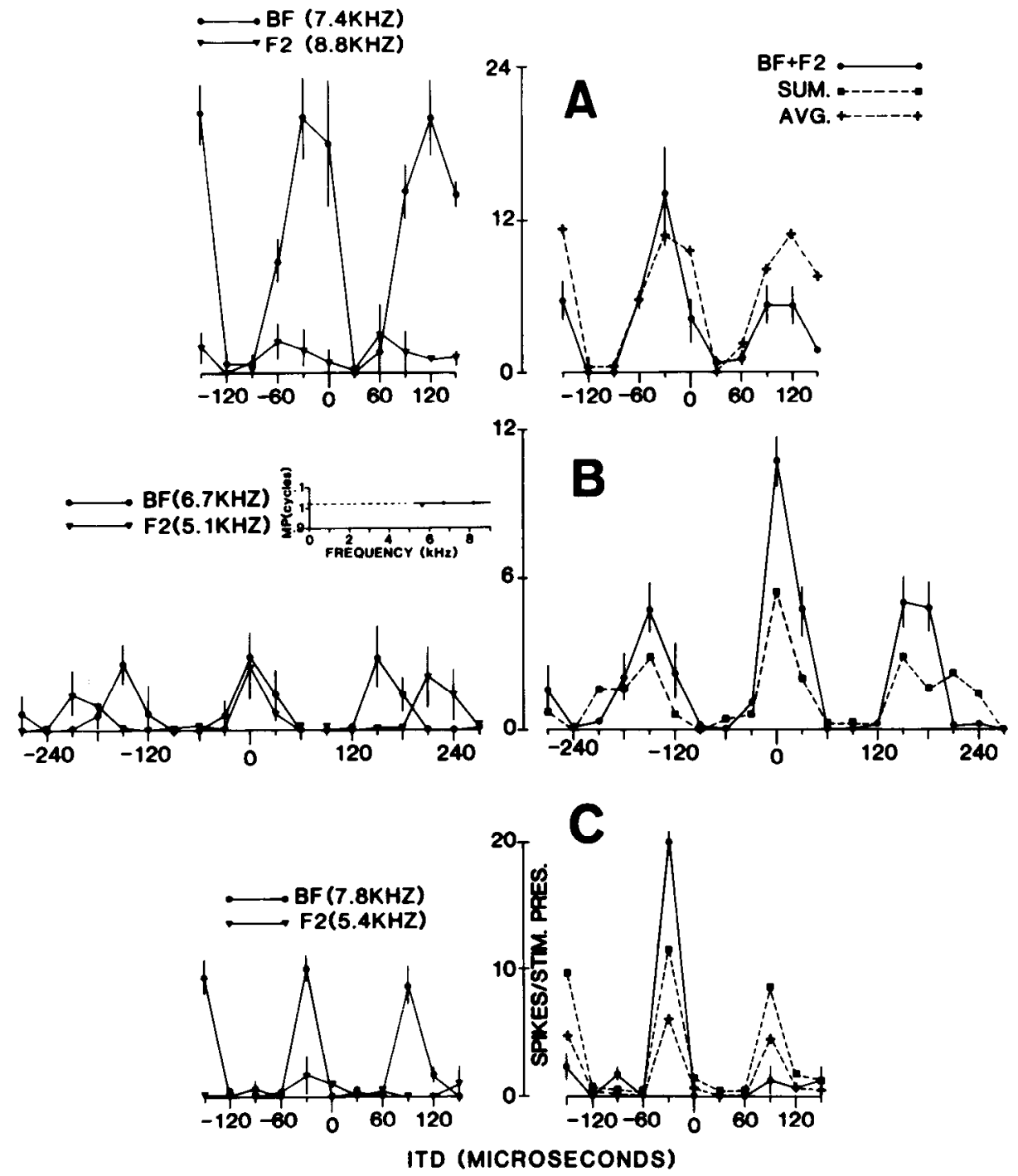

Figure 9. Responses to 2-tone stimuli. The delay curves in the left column were obtained by stimulation with tone bursts at the BF (filled circles) or another frequency, F2 (inverted triangles). The delay curves obtained with 2-tone stimuli are shown in the right column (BF + F2, solid line). The delay curves obtained by summing (dashed line with squares) and/or averaging (dashed line with crosses) the BF and F2 delay curves shown in the left-hand column are superimposed on the 2-tone delay curves for comparison. The levels of the BF and F2, presented as single tones, were equal. The levels of the BF and F2 components in 2-tone stimuli were equal and were also equal to the levels of the BF and F2 when presented as single tones. The neuron shown in $A$ (right external nucleus) depicts an example of secondary peak suppression. The levels of BF burst (7400 $\mathrm{Hz}$ ) and F2 burst $(8800 \mathrm{~Hz})$ were $46 \mathrm{~dB}$ SPL in each ear. The spectral levels of BF and F2 components in 2-tone stimulus were $46 \mathrm{~dB}$ SPL in each ear. The neuron shown in $B$ (left external nucleus) exemplifies facilitation at the $\mathrm{CD}$ (see phase-frequency plot in inset; slope $=0.5 \mu$ sec, $y$-intercept $=0.02$ cycles, MSE $=0.00004, p<0.05)$. The level of BF burst $(6667 \mathrm{~Hz})$ and F2 burst $(5100 \mathrm{~Hz})$ was $56 \mathrm{~dB}$ SPL in the left ear and $54 \mathrm{~dB}$ SPL in the right ear. The spectral level of BF and F2 components in 2-tone stimuli was $56 \mathrm{~dB}$ SPL in the left ear and $54 \mathrm{~dB}$ SPL in the right ear. The neuron in $C$ (right external nucleus) shows an example of primary peak facilitation and secondary peak suppression. The levels of BF burst (7800 $\mathrm{Hz})$ and F2 burst $(5400 \mathrm{~Hz})$ were $46 \mathrm{~dB}$ SPL in each ear. The spectral level of BF and F2 components in 2 -tone stimuli was $46 \mathrm{~dB}$ SPL in each ear. The suppression and facilitation obtained by stimulating with the 2-tone complex cannot be explained by the linear summation or averaging of the BF and F2 delay curves.

\section{Effects of spectral level}

The tone and noise stimuli presented to the cells shown in Figures 2 and 7 were matched for RMS levels. Under these conditions the sound energy is concentrated in a narrow frequency band for tone bursts but is distributed over the entire spectrum for noise bursts. Suppression and facilitation could also be observed when tone and noise bursts were matched for the spectral level of a particular frequency band ( $n=8$ neurons), as shown in Figure 8 . The delay curves shown in Figure $8 A$ were obtained with either the BF alone or with noise plus BF. The level of the BF component in the noise burst was thus equal to the level of the BF presented as a tone burst. As can be seen, the noise/BF combination at $-30 \mu \mathrm{sec}$, which is near the $\mathrm{CD}$ $(-32.2 \mu \mathrm{sec}$, see inset; mean ITD of primary peak $=-35.9$ $\mu \mathrm{sec}$ ), elicited a discharge level 1.7 times that of the BF alone. The response at $+120 \mu \mathrm{sec}$, when the stimulus was the noise/ $\mathrm{BF}$ combination, was roughly half the response to the $\mathrm{BF}$ alone. At +240 and $-180 \mu \mathrm{sec}$ the presence of noise had relatively little effect on the response to the BF.

In Figure $8 B$, the RMS level of the noise was increased so that the level of the band (width $=24.4 \mathrm{~Hz}$ ) centered on the neuron's BF matched the level of a tone burst at the same frequency. With noise stimuli the primary peak is triple the response to the $\mathrm{BF}$ at the corresponding ITD. The response to 
noise at other ITDs is considerably less when compared with the response to the BF at the same ITDs.

\section{Response to 2-tone stimuli: nonlinear interactions}

By stimulating with the summed waveform of the BF and another frequency, $F 2$, we were able to demonstrate interactions between frequency channels that could account for the effects of noise in 44 of 49 neurons tested. Figure 9 illustrates the responses of 3 space-specific neurons to such 2 -tone bursts. The left-hand column in Figure 9 shows the responses of the neurons to their $\mathrm{BF}$ alone and to $\mathrm{F} 2$ alone. The $\mathrm{BF}$ and $\mathrm{F} 2$ were of equal levels in all cases. The right-hand column depicts delay curves obtained with a 2-tone stimulus. The spectral levels of the BF and $\mathrm{F} 2$ in the 2-tone stimulus were equal. Moreover, the spectral levels of the $\mathrm{BF}$ and $\mathrm{F} 2$ in a 2-tone stimulus were equal to the spectral levels of the BF and F2 presented individually. In Figure $9 A$, the $\mathrm{BF}$, presented alone, evoked 20 spikes/stimulus presentation at $-30 \mu \mathrm{sec}$, which is the ITD of the primary peak (response to noise not shown) and at $+120 \mu \mathrm{sec}$. F2 alone was far less excitatory, eliciting 2-4 spikes at -60 and $+60 \mu$ sec. When the summed waveform of the BF and F2 was presented (Fig. $9 A$, right $)$, the discharge at the ITD of the secondary peak $(+90 /$ $+120 \mu \mathrm{sec}$ ) was reduced by $72 \%$ (from 20 to 5.6 spikes/stimulus presentation). A less dramatic reduction in discharge was also observed at $-30 \mu \mathrm{sec}$. Figure $9 B$ illustrates a neuron that exhibited facilitation. This neuron responded poorly to tonal stimuli, discharging at similar, low levels to a number of different frequencies. Consequently, a clear BF could not be established. However, noting that the period of its noise delay curve (not shown) was roughly $150 \mu \mathrm{sec}$, we chose $6667 \mathrm{~Hz}$ (period $=150$ $\mu \mathrm{sec}$ ) as the "BF." It can be seen from Figure $9 B$ (left) that this frequency, as well as $5100 \mathrm{~Hz}$ (F2), clicitcd about 3 spikes/ stimulus presentation at the $\mathrm{CD}(-0.5 \mu \mathrm{sec}$; inset, Fig. $9 B)$ and at the ITD of their respective secondary peaks $( \pm 150 \mu \mathrm{sec}$ for the BF; $\pm 210 \mu \mathrm{sec}$ for F2). Presentation of the 2-tone burst resulted in a facilitation of the response at the $\mathrm{CD}$ (to 10.6 spikes/ stimulus prescntation) and a lesser facilitation at the ITD of the secondary peaks (to 5-6 spikes/stimulus presentation). Both suppression and facilitation are demonstrated in Figure $9 C$. When stimulated with its $\mathrm{BF}(7800 \mathrm{~Hz})$, the neuron responded maximally (8-10 spikes/stimulus presentation) at $-30,-150$, and $190 \mu \mathrm{sec}$ (Fig. 9C, left). F2 $(5400 \mathrm{~Hz})$ was barely effective, evoking, at best, 2.7 spikes/stimulus. When stimulated with the summed waveform, its response at $-30 \mu \mathrm{sec}$ was double (20 spikes/stimulus presentation) that elicited by the BF alone, and the responses at -150 and $+90 \mu \mathrm{sec}$, where secondary peaks would be expected, were, at best, only $20 \%$ ( 2 spikes/stimulus presentation) that elicited by the BF alone. Thus, a single F2 can have both facilitative and suppressive influences, depending on the ITD.

The effects of the summed waveform cannot be explained by a linear summation and/or averaging of the individual tone delay curves. This can be seen in the right-hand column of Figure 9 , which compares the 2-tone delay curve with the curve derived by the summing and/or averaging of the 2 single-tone delay curves shown to the left. For instance, the suppression of the secondary peaks shown in Figure $9 A$ (right) cannot be achieved by the averaging of the $\mathrm{BF}$ and $\mathrm{F} 2$ delay curves (Fig. $9 A$, right, dashed line), nor can the level of enhancement of the CD peak, shown in Figure $9 B$ (right), be attained by the summing of the $\mathrm{BF}$ and F2 delay curves (Fig. 9B, right, dashed line). Similarly, neither summing nor averaging will yield the level of response observed in the neuron of Figure $9 C$ with 2-tone stimuli (Fig. $9 C$, right, dashed lines). Suppressive and facilitative effects therefore appear to involve nonlinear processes.

The suppression of the response at ITDs of the side peaks is not necessarily due to a simple inhibition of 1 frequency channel by another. This is best seen in Figure $9 A$. As is typical of space- specific neurons (Knudsen and Konishi, 1978), this neuron did not spike in the absence of sound stimuli. Thus the discharge observed upon presentation of the $8800 \mathrm{~Hz}$ tone (F2) represented a real, excitatory response, albeit a weak one. Yet, when the $8800 \mathrm{~Hz}$ tone was summed and presented with the BF (7400 $\mathrm{Hz})$, the response at the ITDs of the secondary peaks $(-150$ and $+120 \mu \mathrm{sec}$ ) was significantly lower than the level elicited by the $\mathrm{BF}$ alone. Thus, it was the combination of the frequencies that evoked a suppressive influence. Because spontaneous activity is essentially 0 in space-specific neurons, we were unable to judge whether all suppressive effects are of this nature or whether simple inhibition, detectable by the diminution of spontaneous activity, also plays a role in suppression.

Not all values of F2 evoked the facilitative and suppressive influences described above. Some F2 values had no effect or suppressed or facilitated the responses at the ITDs of the secondary and primary peaks to similar degrees. Moreover, we noted that the facilitative and suppressive $F 2$ values were not necessarily harmonics of the BF of a neuron. This is shown in Figure 9. The systematic relationship between the $\mathrm{BF}$ and the F2s that evoke a facilitative or suppressive discharge appears to be complex and requires further investigation.

\section{Discussion}

The results of the present study suggest that space-specific neurons can signal $C D$ by virtue of interactions between frequency channels. When the stimulus is a tone, space-specific neurons yield cyclic delay curves having the period of the stimulus. When different frequencies are presented, the response period changes, but an ITD usually evokes a maximal response regardless of the frequency, i.e., space-specific neurons have CDs. With noise, there is a single primary peak at or near the $\mathrm{CD}$, and there are smaller, secondary peaks at ITDs that differ from the ITD of the primary peak by integral multiples of the period of the neuron's BF. These observations suggest that although spacespecific neurons have BFs, other frequency bands contribute so as to facilitatc the response at the CD or to suppress the response at non-CDs. Facilitation and suppression are observed not only with noise but with the summed waveform of 2 tones. Responses of 2-tone stimuli could not be explained by an averaging or summing of 2 individual tone delay curves, suggesting that nonlinear neural processes arc involved.

The properties of space-specific neurons described here parallel certain aspects of the barn owl's sound localization behavior. When presented with a speaker broadcasting a tone, the owl makes localization errors that suggest it cannot determine the ear in which the phase of the tone leads (or lags) (Knudscn and Konishi, 1979). Analogously, the space-specific neuron, when stimulated by tones, responds maximally to the CD and to ITDs that differ from the $\mathrm{CD}$ by 1 period of the stimulus tone. When presented with sounds having wide spectra, the owl localizes only the source, and the space-specific neuron responds maximally at or near the CD. It is not known whether the owl can localize, unambiguously and without continuous searching, sources broadcasting sounds comprising only 2 frequencies.

Facilitation and suppression, which allow the space-specific neuron to respond preferentially to the $C D$, may in part be explained if delay curves consist of excitatory peaks flanked by inhibitory troughs. Moiseff (1985) has demonstrated ITD-dependent inhibitory postsynaptic potentials, indicating that spacespecific neurons are inhibited at noneffective ITD values. At the $\mathrm{CD}$, a space-specific neuron can be driven by many frequency bands, and the contributions of each band can facilitate the response of the neuron beyond the level evoked by the BF alone. At other delays, secondary peaks resulting from 1 frequency band may overlap with the inhibitory trough resulting from another frequency band and thus cancel out. Frequencies that are far from the BF would be expected to be effective for suppres- 
sion. A more complex type of inhibitory process is also involved in secondary peak suppression. Specifically, a frequency that is excitatory when presented alone can be suppressive when paired with a neuron's BF. This implies that frequencies that are close to the BF may also be effective suppressors.

The latter type of inhibitory process bears a superficial resemblance to 2-tone suppression at the auditory periphery. It has been shown that 2 tones, each of which is excitatory when presented individually, can suppress the discharge rate or synchrony of single 8th nerve fibers if presented together (Hind et al., 1967; Javel, 1979, 1981; Sachs and Kiang, 1968). Peripheral 2-tone suppression, which is thought to be due to the nonlinearities of the basilar membrane, is probably not involved in the suppression of secondary peaks reported above. In our experimental paradigm, the proportion of BF to $F 2$ is the same (unity) in both ears. We would expect that the amount of peripheral 2-tone suppression (assuming it occurs in the barn owl) would be equal on the 2 sides. Neurons of nucleus laminaris, which compare binaural phase (Sullivan and Konishi, 1986), might show a diminution in overall discharge at all ITDs. This decrease in discharge could be reflected at the level of the spacespecific neuron. The neuron of Figure $9 A$, whose overall response to 2-tone stimuli is less than its overall response to a $\mathrm{BF}$ tone burst, may be an example of such an effect. However, the selective suppression of secondary peaks, which is also observed in Figure $9 A$, would not be expected. Although one might further propose that the thresholds at secondary peaks are for some reason higher, measurement of thresholds of excitation by single tones at ITDs of the primary and secondary peaks revealed no differences (T. Takahashi and M. Konishi, unpublished observations).

Selectivity for a single ITD probably emerges between the external and central nuclei of the inferior colliculus. The central nucleus of the inferior colliculus is the sole and immediate source of afferents to the external nucleus (Knudsen and Knudsen, 1983). In a recent study (Wagner et al., 1986) neurons in the central nucleus, when stimulated with noise, were shown to discharge maximally at ITDs that differ by 1 period of the neuron's BF. This is consistent with the observation that these neurons receive their inputs from only a single, narrow frequency band (see also Geisler et al., 1969), and it suggests that facilitation and suppression between frequencies in the bandwidth of these neurons do not occur. The BF of neurons increases as a function of depth in the central nucleus of the inferior colliculus (Knudsen and Konishi, 1978), and the period of noise delay curves decreases correspondingly. Importantly, however, one ITD is cqually effective for all neurons encountered in a vertical electrode penetration, regardless of BF (Wagner et al., 1986). Furthermore, the mean interaural phase of the neurons was observed to change linearly with BF. Thus, a CD may be assigned to a vertical array of neurons in the central nucleus. HRP retrograde transport showed that neurons in a restricted locus in the external nucleus of the inferior colliculus are innervated by an array in the central nucleus having the same CD. The convergence of the projections from neurons of a tonotopic column may endow space-specific neurons with their broad bandwidth (Knudsen, 1984). The facilitatory and inhibitory interactions described above may occur between the converging frequency bands and allow the space-specific neuron to signal a single ITD.

In the optic tectum, a recipient of afferents from the external nucleus of the inferior colliculus (Knudsen and Knudsen, 1983), neurons have characteristics similar to those described for the space-specific neuron. Using free-field stimuli, Esterly (1984) has shown that tectal neurons have 1 receptive field, the "primary field," when the stimulus is broad-band noise. With narrow-band (one-third octave filtered) noise or tone bursts, "ac- cessory" fields appear at loci that depend upon the stimulus frequency. These results are analogous to our finding that spacespecific neurons are selective for a single ITD when the stimulus is a noise burst but respond equally to multiple ITDs that differ by the period of the stimulus tone when the stimulus is a tone burst. Esterly (1984) further reports that adding a second frequency to the narrow-band noise suppresses the tectal neuron's response when the source is in an accessory field and facilitates the response when the source is in the primary field. These findings are analogous to our 2-tone experiments, wherein the response of space-specific neurons can be suppressed at the ITD of a secondary peak and facilitated at the ITD of the primary peak by the addition of F2. Thus our present observations, which were made using diotic stimulation, have analogies in experiments that implement free-field stimulation. It is likely that the characteristics observed in the optic tectum reflect those of its afferent source, the external nucleus of the inferior colliculus.

\section{Comparisons with studies in mammals}

\section{Interaural delay sensitivity}

Facilitation and inhibition that occur as a function of interaural delay of single tones are well-established phenomena in the mammalian auditory system (Brugge et al., 1970; Goldberg and Brown, 1969; Kuwada and Yin, 1983; Kuwada et al., 1984; Rose et al., 1966; Yin and Kuwada, 1983). At favorable delays discharge levels are enhanced beyond the sum of monaural discharge levels, and at other delays discharge is less than monaural levels. Thus, as suggested above for the space-specific neuron, delay functions obtained from mammalian auditory neurons often have peaks of facilitation separated by troughs of inhibition. Given such delay curves, it would not be surprising if suppression of secondary peaks and facilitation of $C D$ peaks were to be observed in mammalian auditory neurons upon presentation of 2-tone stimuli. Two secondary peaks can fall into each other's inhibitory trough and thus mutually cancel, provided that the frequencies are sufficiently different. At the $C D$, peaks can coincide and facilitation occur, provided that 1 frequency does not drive the neuron to saturation. Unfortunately, 2 -tone stimuli are not commonly employed in the study of binaural response, and data are unavailable. Crow et al. (1980) used noise and 2-tone complexes but did not compare the results obtained with these stimuli to those obtained with a neuron's BF. Moreover, the $200 \mu$ sec steps with which they sampled the ITD selectivity of high-frequency neurons (above $3 \mathrm{kHz}$ ) are too coarse to rcsolve multiple peaks and judge their magnitudes.

The effects of noise have been investigated more extensively, but the findings suggest that facilitation and suppression between frequency bands do not occur. Geisler et al. (1969) stimulated neurons from the cat inferior colliculus with wide-spectrum noise and demonstrated that delay-responsc curves have periods equivalent to that of the characteristic frequency. Interestingly, at least 1 neuron that they illustrated (their Fig. 1) showed peaks of roughly equal height at a number of delays, indicating a lack of the type of interfrequency band interactions described for the space-specific neuron. Recent studies show that noise delay curves of neurons in the cat inferior colliculus (Chan et al., 1983) and medial superior olive (Chan and Yin, 1984) closely resemble curves generated by the averaging of delay curves obtained with the tones that compose the noise ("composite curves"). Thus, neurons of the cat's medial superior olive and inferior colliculus sum the contributions of different frequency bands. Because the cat relies on interaural phase differences of lower frequencies, secondary peaks generally fall outside the physiologically relevant range. Mechanisms to suppress secondary peaks would not be necessary. 
The $C D$

The CD was originally defined by Rose et al. (1966) as "the (interaural) delay at which the periodic discharge curves for different frequencies reach the same relative amplitude." Thus, CD can be represented by maximal, minimal, or intermediate levels of spike activity. Our results suggest that space-specific neurons generally respond at or near maximal levels at the CD. That space-specific neurons signal the $C D$ delay with a maximal response is not surprising. It has already been shown that spacespecific neurons are driven maximally from a circumscribed location in space (i.e., they have spatial receptive fields) (Knudsen and Konishi, 1978), and CD is directly related to the direction from which a sound originates. Furthermore, Moiseff and Konishi (1981) have shown that the ITD that evokes the greatest discharge in a neuron and the azimuthal center of the neuron's spatial receptive field are strongly correlated. Although the authors did not show that their most effective ITD was the $\mathrm{CD}$, they did use noise, and the results of the present study suggests that, with noise, space-specific neurons discharge maximally at the CD.

Yin and Kuwada (1983) have demonstrated that neurons of the central nucleus of the cat's inferior colliculus have CDs at peaks, troughs, or intermediate levels. In fact, most of their neurons express the $\mathrm{CD}$ at an intermediate discharge level. Yin and Kuwada (1983) suggest that other parameters of a neuron's activity may be more relevant to the encoding of sound source locations than are CD or CP. Because unilateral ablations of the inferior colliculus in the cat leads to a predominantly contralateral deficit (Jenkins and Masterton, 1982), one would expect a contralateral bias in the distribution of the parameter that encodes space. Yin and Kuwada (1983) observed the expected contralatcral bias not in the distribution of the CDs or CPs of their cells but in the distributions of the peaks and troughs of their composite delay curves (obtained by averaging a neuron's tone delay curves). They point out, moreover, that the mean interaural phase difference of some of their neurons varied with sound pressure level, which could cause the CDs of the neurons to be dependent on sound pressure level. The positions of the peaks and troughs of composite curves, by comparison, were level-tolerant. Thus, the authors speculated that the maxima and minima of composite curves are the relevant parameters in coding sound source location. In addition to the peaks and troughs, they also suggest that the slopes of composite curves may form a useful code, were the source to move with respect to the cat's head. Studies combining diotic and free-field stimuli and further studies of feline sound localization behavior are required to evaluate these mechanisms.

Space-specific neurons are arrayed in the external nucleus of the inferior colliculus according to the locations of their receptive fields so as to form a map that is topologically isomorphic with external space (Knudsen and Konishi, 1978). Because a similar map has been demonstrated in the superior colliculus of the cat (Middlebrooks and Knudsen, 1984), there is the possibility that the external nucleus of the cat's inferior colliculus, a source of efferents to its superior colliculus (Kudo and Niimi, 1980), contains neurons with interaural delay responses similar to those of the space-specific neuron. Although direction-sensitive and direction-selective neurons have been demonstrated in the external nucleus of the cat's inferior colliculus (Aitkin et al., 1984), their binaural properties are not known.

\section{References}

Aitkin, L. M., G. R. Gates, and S. C. Philips (1984) Responses of neurons in inferior colliculus to variations in sound-source azimuth. J. Neurophysiol. 52: 1-17.

Brugge, J. F., D. J. Anderson, and L. M. Aitkin (1970) Responses of neurons in the dorsal nucleus of the lateral lemniscus of cat to binaural tonal stimulation. J. Neurophysiol. 33: 441-458.

Chan, J. C. K., and T. C. T. Yin (1984) Interaural time sensitivity in the medial superior olive of the cat: Comparison with the inferior colliculus. Soc. Neurosci. Abstr. 10: 844.

Chan, J. C. K., D. R. F. Irvine, A. D. Musicant, and T. C. T. Yin (1983) Comparison of responses to interaurally delayed low-frequency tonal and wide band noise stimuli in the cat's inferior colliculus. Soc. Neurosci. Abstr. 9: 766

Crow, G., T. L. Langford, and G. Moushegian (1980) Coding of interaural time differences by some high-frequency neurons of the inferior colliculus: Responses to noise bands and two-tone complexes. Hearing Res. 3: 147-153.

Esterly, S. E. (1984) Responses of space-specific neurons in the optic tectum of the owl to narrow band sounds. Soc. Neurosci. Abstr. 30: 1149.

Geisler, C. D., W. S. Rhode, and D. W. Hazelton (1969) Responses of inferior colliculus neurons in the cat to binaural acoustic stimuli having wide band spectra. J. Neurophysiol. 32: 960-974.

Goldberg, J. M., and P. B. Brown (1969) Response of binaural neurons of dog superior olivary complex to dichotic tonal stimuli: Some physiological mechanisms of sound localization. J. Neurophysiol. 32:613636

Hind, J. E., D. J. Anderson, J. F. Brugge, and J. E. Rose (1967) Coding of information pertaining to paired low-frequency tones in single auditory nerve fibers. J. Neurophysiol. 30: 794-816.

Javel, E. (1979) Two-tone suppression in the auditory nerve of the cat. Effects of excitatory F2. J. Acoust. Soc. Am. [Suppl. 1] 64: S1 35.

Javel, E. (1981) Suppression of auditory nerve responses. I: Temporal analysis, intensity effects, and suppression contours. J. Acoust. Soc. Am. 69: 1735-1745.

Jenkins, W. M., and R. B. Masterton (1982) Sound localization: Effects of unilateral lesions in central auditory system. J. Neurophysiol. 47: 987-1016

Knudsen, E. I. (1983) Subdivisions of the inferior colliculus in the barn owl (Tyto alba). J. Comp. Neurol. 218: 174-186.

Knudsen, E. I. (1984) Synthesis of a neural map of auditory space. In Dynamic Aspects of Neocortical Function, G. M. Edelman, W. M. Cowan, and W. E. Gall, eds., pp. 375-396, Wiley, New York.

Knudsen, E. I., and P. F. Knudsen (1983) Space-mapped auditory projections from the inferior colliculus to the optic tectum in the barn owl (Tyto alba). J. Comp. Neurol. 218: 187-196.

Knudsen, E. I., and M. Konishi (1978) Space and frequency are represented separately in auditory midbrain of the owl. J. Neurophysiol. 41: 870-884.

Knudsen, E. I., and M. Konishi (1979) Mechanisms of sound localization in the barn owl (Tyto alba). J. Comp. Physiol. 133: 13-21.

Konishi, M. (1973) How the owl tracks its prey. Am. Sci. 61: 414 424.

Kudo, M., and K. Niimi (1980) Ascending projections of the inferior colliculus. J. Comp. Neurol. 191: 545-556.

Kuwada, S., and T. C. T. Yin (1983) Binaural interactions in lowfrequency neurons in inferior colliculus of cat. I. Effects of long interaural delays, intensity, and repetition rate on interaural delay function. J. Neurophysiol. 50:981-999.

Kuwada, S., T. C. T. Yin, J. Syka, T. J. F. Buunen, and R. E. Wickesberg (1984) Binaural interactions in low-frequency neurons in the inferior colliculus of cat. IV. Comparison of monaural and binaural response properties. J. Neurophysiol. 51: 1306-1325.

Middlebrooks, J. C., and E. I. Knudsen (1984) A neural code for auditory space in the cat's superior colliculus. J. Neurosci. 4: 26212634

Mills, A. W. (1972) Auditory localization. In Foundations of Modern Auditory Theory, Vol. 2, J. V. Tobias, ed., Academic, New York.

Moiseff, A. (1985) Intracellular recordings from the inferior colliculus. Soc. Neurosci. Abstr. 11: 735.

Moiseff, A., and M. Konishi (1981) Neuronal and behavioral sensitivity to binaural time difference in the owl. J. Neurosci. 1: 40-48.

Moiseff, A., and M. Konishi (1983) Binaural characteristics of units in the owl's brainstem auditory pathway: Precursors of restricted spatial receptive fields. J. Neurosci. 3: 2553-2562.

Moiseff, A., and M. Konishi (1984) Localization of virtual sound images by barn owls. Soc. Neurosci. Abstr. 10: 1150 .

Rose, J. E., N. B. Gross, C. D. Geisler, and J. E. Hind (1966) Some neural mechanisms in the inferior colliculus of the cat which may be 
relevant to localization of a sound source. J. Neurophysiol. 29: 288314.

Sachs, M. B., and N. Y. S. Kiang (1968) Two-tone inhibition in auditory nerve fibers. J. Acoust. Soc. Am. 43: 1120-1 128.

Sullivan, W. E., and M. Konishi (1986) A map of interaural phase difference in the owl. Proc. Nat. Acad. Sci. USA (in press).

Takahashi, T., A. Moiseff, and M. Konishi (1984) Time and intensity cues are processed independently in the auditory system of the owl. J. Neurosci. 4: 1781-1786.
Wagner, H., T. Takahashi, and M. Konishi (1986) The central nucleus of the inferior colliculus as an input stage to the map of auditory space in the barn owl. Abst. Assoc. Res. Otolaryngol., p. 44.

Yin, T. C. T., and S. Kuwada (1983) Binaural interaction in lowfrequency neurons of inferior colliculus of cat. III. Effects of changing frequency. J. Neurophysiol. 50: 1020-1042. 